\title{
Heisenberg antiferromagnet on the kagome lattice with arbitrary spin: A higher-order coupled cluster treatment
}

\author{
O. Götze, ${ }^{1}$ D. J. J. Farnell, ${ }^{2}$ R. F. Bishop, ${ }^{3}$ P. H. Y. Li,${ }^{3}$ and J. Richter ${ }^{1}$ \\ ${ }^{1}$ Institut für Theoretische Physik, Otto-von-Guericke Universität Magdeburg, D-39016 Magdeburg, Germany \\ ${ }^{2}$ Division of Mathematics and Statistics, Faculty of Advanced Technology, University of Glamorgan, \\ Pontypridd CF37 1DL, Wales, United Kingdom \\ ${ }^{3}$ School of Physics and Astronomy, The University of Manchester, Manchester M13 9PL, United Kingdom
}

(Received 14 October 2011; published 27 December 2011)

\begin{abstract}
Starting with the $\sqrt{3} \times \sqrt{3}$ and the $q=0$ states as reference states, we use the coupled cluster method to high orders of approximation to investigate the ground state of the Heisenberg antiferromagnet on the kagome lattice for spin quantum numbers $s=1 / 2,1,3 / 2,2,5 / 2$, and 3 . Our data for the ground-state energy for $s=1 / 2$ are in good agreement with recent large-scale density-matrix renormalization group and exact diagonalization data. We find that the ground-state selection depends on the spin quantum number $s$. While for the extreme quantum case, $s=1 / 2$, the $q=0$ state is energetically favored by quantum fluctuations, for any $s>1 / 2$ the $\sqrt{3} \times \sqrt{3}$ state is selected. For both the $\sqrt{3} \times \sqrt{3}$ and the $q=0$ states the magnetic order is strongly suppressed by quantum fluctuations. Within our coupled cluster method we get vanishing values for the order parameter (sublattice magnetization) $M$ for $s=1 / 2$ and $s=1$, but (small) nonzero values for $M$ for $s>1$. Using the data for the ground-state energy and the order parameter for $s=3 / 2,2,5 / 2$, and 3 we also estimate the leading quantum corrections to the classical values.
\end{abstract}

DOI: 10.1103/PhysRevB.84.224428

PACS number(s): 75.10.Jm, 75.10.Kt, 75.50.Ee

\section{INTRODUCTION}

The investigation of the low-energy physics of the Heisenberg antiferromagnet (HAFM)

$$
H=\sum_{\langle i, j\rangle} \mathbf{s}_{i} \cdot \mathbf{s}_{j}
$$

on the kagome lattice is one of the most challenging problems in the field of frustrated quantum magnetism. The sum over $\langle i, j\rangle$ runs over all nearest-neighbor pairs of sites on the lattice, counting each bond once only, and $\mathbf{s}_{i} \equiv\left(s_{i}^{x}, s_{i}^{y}, s_{i}^{z}\right)$ is the spin operator on site $i$. Although, there has been an intensive discussion of the problem over many years applying various theoretical methods (see, e.g., Refs. 1-34), no conclusive answer on the nature of the ground state (GS) and the existence of a spin gap has been found.

While for many years a spin-liquid GS was favored, ${ }^{5,10,12}$ recently arguments have been given for a valence-bond crystal GS with a large unit cell of 36 sites that breaks the symmetry of the underlying kagome lattice. ${ }^{16,20,21}$ However, very recently this valence-bond picture has been rechecked by large-scale numerics ${ }^{28,29,31}$ and once again the spin-liquid GS is favored.

Although large-scale density-matrix renormalization group (DMRG) and exact diagonalization (ED) calculations seem to be most effective to study the low-energy physics of the kagome HAFM, complementary methods (see, e.g., Refs. 27, 30,33 , and 34), are highly desirable to shed further light on this challenging problem.

A method which has been successfully applied to strongly frustrated quantum magnets is the coupled cluster method (CCM) (see, e.g., Refs. 14, 26, and 35-42). In the present paper we apply the CCM in high orders of approximation to the kagome HAFM.

\section{COUPLED CLUSTER METHOD}

For the sake of brevity we illustrate here only some relevant features of the coupled cluster method (CCM). For more general information on the methodology of the CCM, see, e.g., Refs. 43-48.

We first mention that the CCM approach yields results directly in the thermodynamic limit $N \rightarrow \infty$, where $N$ is the number of lattice sites (and hence spins). The starting point for a CCM calculation is the choice of a normalized reference state $|\Phi\rangle$ that is typically a classical GS of the model. For the kagome HAFM we choose the $\sqrt{3} \times \sqrt{3}$ and the $q=0$ states illustrated in Fig. 1 (see also, e.g., Refs. 2, 7, and 33 for further details). Then we perform a rotation of the local axes of each of the spins such that all spins in the reference state align along the negative $z$ axis. In this new set of local spin coordinates a complete set of mutually commuting multispin creation operators $C_{I}^{+} \equiv\left(C_{I}^{-}\right)^{\dagger}$ related to this reference state is defined by

$$
|\Phi\rangle=|\downarrow \downarrow \downarrow \cdots\rangle, \quad C_{I}^{+}=s_{n}^{+}, s_{n}^{+} s_{m}^{+}, s_{n}^{+} s_{m}^{+} s_{k}^{+}, \ldots,
$$

where $s_{n}^{+} \equiv s_{n}^{x}+i s_{n}^{y}$, the indices $n, m, k, \ldots$ denote arbitrary lattice sites, and the components of the spin operators are defined in the local rotated coordinate frames. Note that for spins of quantum number $s$, each site index in each configuration index $I$ in Eq. (2) can be repeated up to a maximum of $2 s$ times. With the set $\left\{|\Phi\rangle, C_{I}^{+}\right\}$thus defined, the CCM parametrizations of the ket and bra GS eigenvectors $|\Psi\rangle$ and $\langle\tilde{\Psi}|$ of the spin system are given by

$$
\begin{gathered}
|\Psi\rangle=e^{S}|\Phi\rangle, \quad S=\sum_{I \neq 0} a_{I} C_{I}^{+}, \\
\langle\tilde{\Psi}|=\langle\Phi| \tilde{S} e^{-S}, \quad \tilde{S}=1+\sum_{I \neq 0} \tilde{a}_{I} C_{I}^{-} .
\end{gathered}
$$



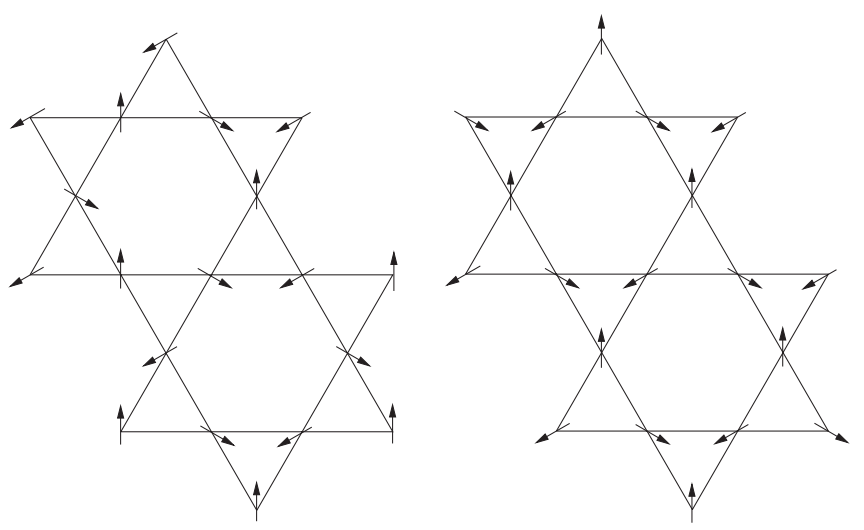

FIG. 1. Illustration of the $\sqrt{3} \times \sqrt{3}$ (left) and the $q=0$ (right) classical GS of the kagome HAFM.

We have defined $C_{0}^{+} \equiv 1$, and the normalization of the states is clearly such that $\langle\tilde{\Psi} \mid \Psi\rangle=\langle\Phi \mid \Psi\rangle=\langle\Phi \mid \Phi\rangle \equiv 1$. The CCM correlation operators $S$ and $\tilde{S}$ contain the correlation coefficients $a_{I}$ and $\tilde{a}_{I}$, which can be determined by the CCM ket-state and bra-state equations

$$
\begin{gathered}
\left\langle\Phi\left|C_{I}^{-} e^{-S} H e^{S}\right| \Phi\right\rangle=0, \quad \forall I \neq 0, \\
\left\langle\Phi\left|\tilde{S} e^{-S}\left[H, C_{I}^{+}\right] e^{S}\right| \Phi\right\rangle=0, \quad \forall I \neq 0 .
\end{gathered}
$$

Equations (5) and (6) are fully equivalent to the GS Schrödinger equations for the ket and bra states. They follow readily from the requirement that the GS energy functional $\langle\tilde{\Psi}|H| \Psi\rangle$ be stationary with respect to variations in all of the correlation coefficients $\tilde{a}_{I}$ and $a_{I}$, respectively $(\forall I \neq 0)$. Each ket-state or bra-state equation belongs to a certain configuration index $I$, i.e., it corresponds to a certain set (configuration) of lattice sites $n, m, k, \ldots$, as in Eq. (2). Using the Schrödinger equation, $H|\Psi\rangle=E|\Psi\rangle$, we can now write the GS energy as $E=\left\langle\Phi\left|e^{-S} H e^{S}\right| \Phi\right\rangle$. The magnetic order parameter (sublattice magnetization) is given by $M=$ $-\frac{1}{N} \sum_{i=1}^{N}\left\langle\tilde{\Psi}\left|s_{i}^{z}\right| \Psi\right\rangle$, where $s_{i}^{z}$ is expressed in the transformed coordinate system, and $N(\rightarrow \infty)$ is the number of lattice sites.

If we would be able to consider all creation and annihilation operators $C_{I}^{+}$and $C_{I}^{-}$, i.e., all sets (configurations) of lattice sites, in the CCM correlation operators $S$ and $\tilde{S}$ we would get, in principle, the exact eigenstate. ${ }^{44}$ However, for the manybody quantum system under consideration it is necessary to use approximation schemes in order to truncate the expansions of $S$ and $\tilde{S}$ in Eqs. (3) and (4) in a practical calculation. Then the approximate results for the GS energy $E$ and the order parameter $M$ will depend certainly on the choice of the reference state.

We use for spin quantum number $s=1 / 2$ the so-called LSUB $n$ approximation scheme to truncate the expansions of $S$ and $\tilde{S}$ in Eqs. (3) and (4), where we include only $n$ or fewer correlated spins in all configurations (or lattice animals in the language of graph theory) which span a range of no more than $n$ contiguous lattice sites, where a set of sites is defined to be contiguous if every site has at least one other in the set as a nearest neighbor (for more details see Refs. 43 and 46-48). Using efficient parallelized CCM $\operatorname{code}^{49}$ we are able to solve the CCM equations up to LSUB10 for $s=1 / 2$ (where, e.g., for the $q=0$ reference state a set of 238010 coupled ket-state equations has to be solved), which goes significantly beyond earlier CCM calculations for the kagome HAFM. ${ }^{14,26}$ Moreover, we also use the CCM to consider spin quantum numbers $s>1 / 2$.

Since the LSUB $n$ approximation becomes exact for $n \rightarrow$ $\infty$ (as does the alternative SUB $n-n$ scheme that we introduce and use in Sec. III B for values of the spin quantum number $s>1 / 2$ ), it is useful to extrapolate the "raw" LSUB $n$ (or SUB $n-n$ ) data to the limit $n \rightarrow \infty$. There is ample experience regarding how one should extrapolate the GS energy per site $e_{0}(n) \equiv E(n) / N$ and the magnetic order parameter $M(n)$. For the GS energy per spin, $e_{0}(n)=a_{0}+a_{1}(1 / n)^{2}+a_{2}(1 / n)^{4}$ is a very well-tested extrapolation ansatz. ${ }^{35-38,40,46,48}$ An appropriate extrapolation rule for the magnetic order parameter of highly frustrated systems is ${ }^{37,38,40} M(n)=b_{0}+b_{1}(1 / n)^{1 / 2}+$ $b_{2}(1 / n)^{3 / 2}$. Moreover, we know from Refs. 37, 38, and 40 that low levels of approximation conform poorly to these rules. Hence, we exclude the $n=2$ and $n=3$ data from the extrapolations.

For the solution of the CCM equations we rewrite the Hamiltonian (1) in the rotated coordination frame of the local quantization axis

$$
\begin{aligned}
H_{\lambda}= & \sum_{\langle i \rightarrow j\rangle}\left(-\frac{1}{2}\left(\lambda s_{i}^{x} s_{j}^{x}+s_{i}^{z} s_{j}^{z}\right)+\lambda s_{i}^{y} s_{j}^{y}\right. \\
& \left.+\frac{\sqrt{3}}{2} \lambda\left(-s_{i}^{x} s_{j}^{z}+s_{i}^{z} s_{j}^{x}\right)\right),
\end{aligned}
$$

where we have further introduced an anisotropy parameter $\lambda$ that now multiplies the non-Ising terms, similar to what was done in Refs. 6 and 45. Note that the symbols $\langle i \rightarrow j\rangle$ on the sum in Eq. (7) now indicate directionality of the nearest-neighbor bonds (see, e.g., Refs. 6, 39, and 45), which is different for the $\sqrt{3} \times \sqrt{3}$ and the $q=0$ reference states. Starting at $\lambda=0$, where the corresponding reference states are eigenstates of $H_{\lambda}$, we can slowly increase $\lambda$ and hence trace the CCM solutions out to the true kagome point at $\lambda=1$. Moreover, $\lambda$ can be understood as a parameter that tunes the strength of the quantum fluctuations.

\section{RESULTS}

\section{A. The extreme quantum case: $s=1 / 2$}

We start with the CCM investigation of the kagome HAFM for spin quantum number $s=1 / 2$. At a given finite level of the CCM LSUB $n$ scheme, the treatment of quantum effects is performed in an approximate manner. Certainly, the treatment of quantum effects becomes better as the level of approximation $n$ is increased. In previous studies of the GS selection based on an expansion around the classical limit, ${ }^{4,7,11}$ the $\sqrt{3} \times \sqrt{3}$ state was found to be selected by quantum fluctuations. We present our results for the GS energy per site in Fig. 2, where the dependence on the anisotropy parameter $\lambda$ of the difference in the energies per site between the two states considered, $\Delta e \equiv e_{0}^{\sqrt{3} \times \sqrt{3}}-e_{0}^{q=0}$, is shown for various LSUB $n$ approximations. Interestingly, the GS selection depends on the LSUB $n$ truncation index $n$. Just as in linear spin-wave theory, ${ }^{2,11}$ there is also no GS selection 


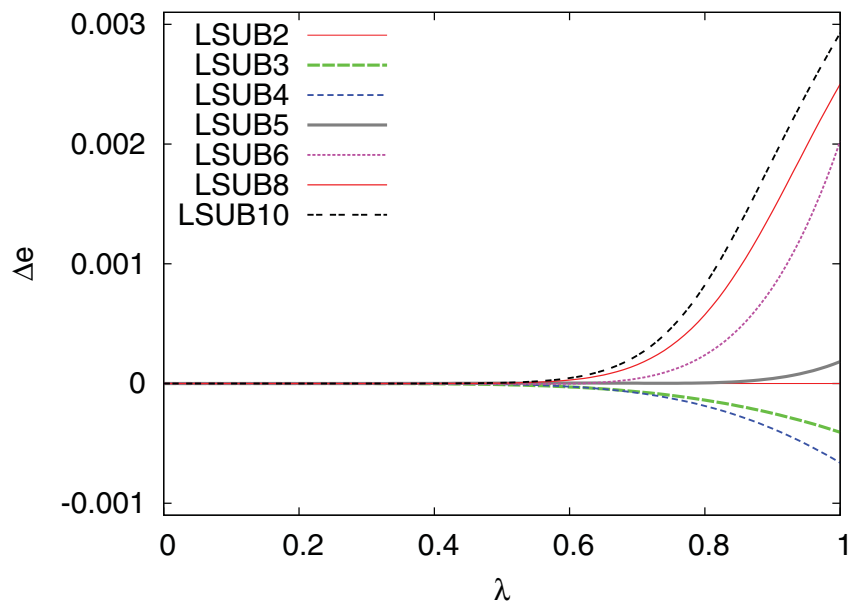

FIG. 2. (Color online) Difference of GS energies per site, $\Delta e \equiv$ $e_{0}^{\sqrt{3} \times \sqrt{3}}-e_{0}^{q=0}$, between the $\sqrt{3} \times \sqrt{3}$ and $q=0$ states of the spin$1 / 2$ kagome HAFM, for various CCM LSUB $n$ approximations and spin quantum number $s=1 / 2$.

(i.e., $\Delta e=0$ ) at the CCM-LSUB2 level, thereby indicating a poor consideration of quantum effects at the lowest LSUBn order. As the level of approximation $n$ is increased we first find

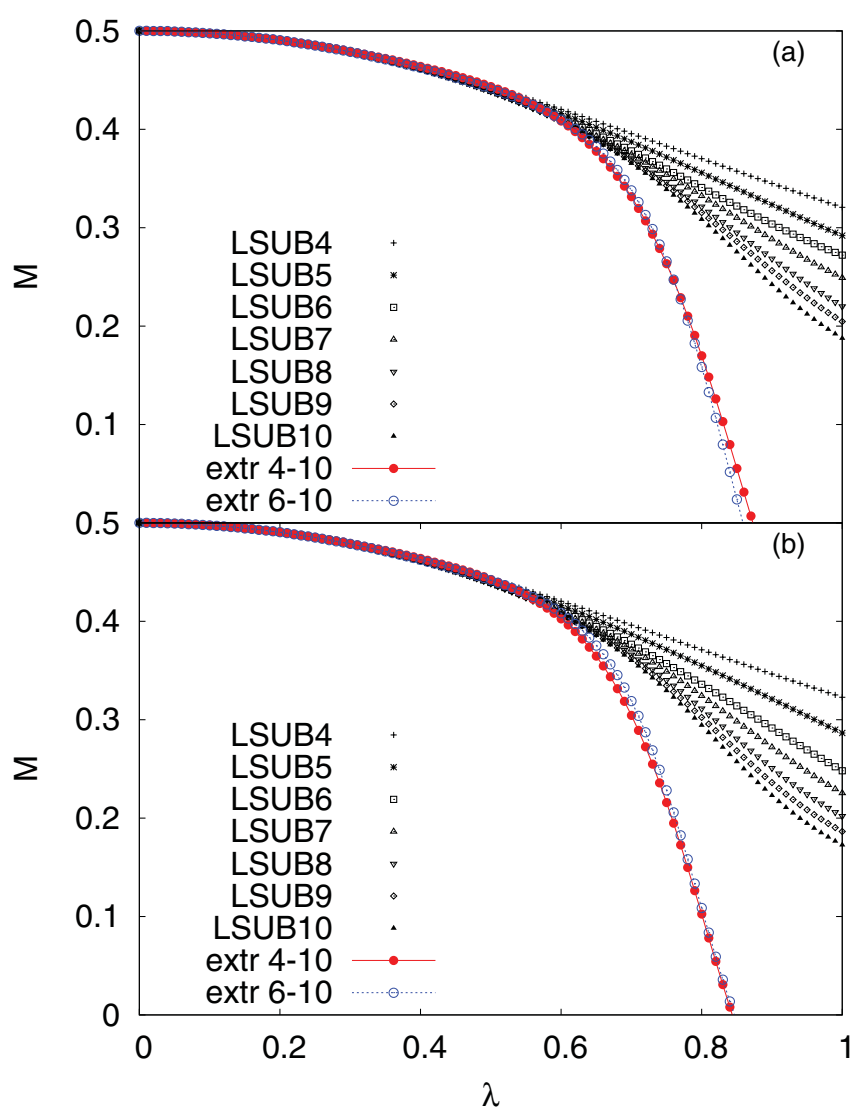

FIG. 3. (Color online) CCM-LSUB $n$ data for the magnetic order parameter $M$ versus $\lambda$ for the spin-1/2 kagome HAFM, for (a) the $\sqrt{3} \times \sqrt{3}$ reference state and (b) the $q=0$ reference state. For the extrapolations to $n \rightarrow \infty$ according to $M(n)=b_{0}+b_{1}(1 / n)^{1 / 2}+$ $b_{2}(1 / n)^{3 / 2}$ we have used LSUB $n$ data for $n=4,5, \ldots, 10$ as well as for $n=6,7, \ldots, 10$. that $\Delta e<0$ for $n=3$ and $n=4$ (i.e., the $\sqrt{3} \times \sqrt{3}$ state is selected in accordance with previous findings ${ }^{7,11}$ ), but as $n$ is further increased we then find that $\Delta e>0$ for $n>4$ (i.e., the $q=0$ state is selected). Bearing in mind that quantum effects are better taken into account at higher LSUB $n$ levels, we might argue that strong quantum fluctuations indeed favor the $q=0$ state. Note that this line of argument is also supported by our CCM results below for spin quantum numbers $s>1 / 2$ (i.e., generally speaking, where quantum fluctuations are weaker), where in all levels of approximations the $\sqrt{3} \times \sqrt{3}$ state is selected (see our discussion below in Sec. III B).

In Fig. 3 we show the magnetic order parameter as a function of the anisotropy parameter $\lambda$. At $\lambda=0$ we have $M=$ $s=1 / 2$, since the corresponding reference state is the exact GS of $H_{\lambda=0}$. As $\lambda$ is increased the order parameter decreases monotonically. At a certain value of $\lambda$, near the true kagome point $\lambda=1$, the extrapolated order parameter vanishes, thus indicating that the GS is magnetically disordered. The difference in the two variants of the extrapolation (including or excluding LSUB4 and LSUB5) may be considered as an error bar for the extrapolated order parameter.

To illustrate the quality of the extrapolations of the "raw" LSUB $n$ data in the limit $n \rightarrow \infty$, we show corresponding plots for the $q=0$ state and various values of the anisotropy parameter $\lambda$ in Fig. 4 . It is obvious that the LSUB $n$ data are well fitted by the applied extrapolation functions.
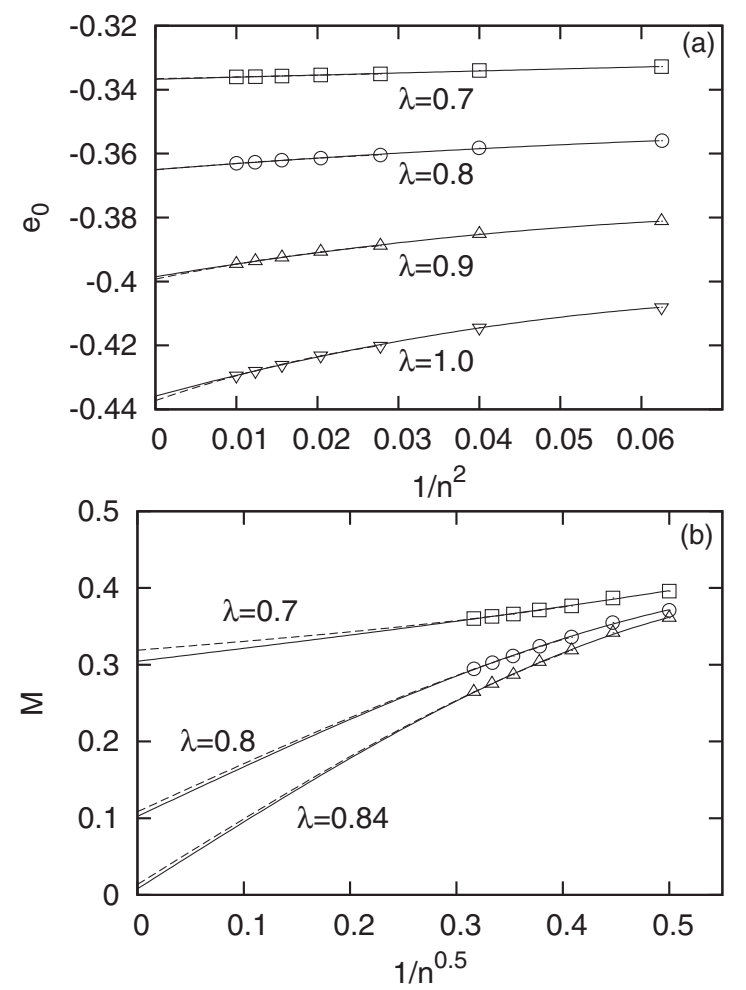

FIG. 4. Illustration of the extrapolation of the CCM-LSUB $n$ data (symbols) for (a) the ground-state energy $e_{0}$ and (b) the magnetic order parameter $M$ for the $q=0$ state of the spin-1/2 kagome HAFM. For the extrapolations to $n \rightarrow \infty$ according to $e_{0}(n)=$ $a_{0}+a_{1}(1 / n)^{2}+a_{2}(1 / n)^{4}$ and $M(n)=b_{0}+b_{1}(1 / n)^{1 / 2}+b_{2}(1 / n)^{3 / 2}$ we have used LSUB $n$ data for $n=4,5, \ldots, 10$ (solid lines) as well as for $n=6,7, \ldots, 10$ (dashed lines). 
TABLE I. CCM results for the spin-1/2 HAFM on the kagome lattice (i.e., at $\lambda=1$ ). The quantity $e_{0} \equiv E / N$ is the GS energy per spin and $M$ is the magnetic order parameter (sublattice magnetization). The LSUB $n$ results are extrapolated to $n \rightarrow \infty$ according to $e_{0}(n)=$ $a_{0}+a_{1}(1 / n)^{2}+a_{2}(1 / n)^{4}$ and $M(n)=b_{0}+b_{1}(1 / n)^{1 / 2}+b_{2}(1 / n)^{3 / 2}$ using LSUB $n$ data for $n=4,5, \ldots, 10$ as well as for $n=6,7, \ldots, 10$.

\begin{tabular}{llc}
\hline \hline$\sqrt{3} \times \sqrt{3}$ & \multicolumn{1}{c}{$e_{0}$} & $M$ \\
\hline LSUB4 & -0.408728 & 0.320702 \\
LSUB5 & -0.414235 & 0.291917 \\
LSUB6 & -0.418052 & 0.272109 \\
LSUB7 & -0.420677 & 0.248989 \\
LSUB8 & -0.423554 & 0.219994 \\
LSUB9 & -0.424962 & 0.204661 \\
LSUB10 & -0.426485 & 0.187634 \\
Extrapolated (4-10) & -0.4318 & $<0$ \\
Extrapolated (6-10) & -0.4336 & $<0$ \\
$q=0$ & $e_{0}$ & $M$ \\
LSUB4 & -0.408066 & 0.322860 \\
LSUB5 & -0.414418 & 0.286462 \\
LSUB6 & -0.420078 & 0.248078 \\
LSUB7 & -0.423126 & 0.225356 \\
LSUB8 & -0.426054 & 0.202074 \\
LSUB9 & -0.427952 & 0.186435 \\
LSUB10 & -0.429413 & 0.172742 \\
Extrapolated (4-10) & -0.4357 & $<0$ \\
Extrapolated (6-10) & -0.4372 & $<0$ \\
Other recent results & & - \\
Ref. 20 & -0.433 & - \\
Ref. 27 & -0.4322 & - \\
Ref. 29, $N=42$ (type a) & -0.437999 & - \\
Ref. 29, $N=42$ (type b) & -0.438143 & -0.4379 \\
Ref. 28 & & \\
\hline \hline
\end{tabular}

Next we analyze the model for $\lambda=1$ in more detail. The CCM-LSUB $n$ data, as well as the extrapolated data, are listed in Table I. Moreover, we present results for the GS energy obtained by other methods for comparison. While the values $e_{0}=-0.4322$ obtained in Ref. 27 and $e_{0}=-0.4332$ obtained in Ref. 28 can be considered as rigorous upper bounds of the GS energy, the large-scale DMRG result $e_{0}=-0.4379$ obtained in Ref. 28 seems to be the most accurate estimate presently available. The lowest extrapolated CCM energy is $e_{0}=-0.4372$, obtained for the $q=0$ reference state using CCM-LSUB $n$ results for $n=6,7,8,9$, and 10 for the extrapolation. This CCM estimate is very close to the DMRG result of Ref. 28.

\section{B. Higher spin quantum numbers: $s>1 / 2$}

Although several magnetic kagome compounds carry spins with $s>1 / 2, \quad$ such as the $s=3 / 2$ magnet $\mathrm{KCr}_{3}(\mathrm{OH})_{6}\left(\mathrm{SO}_{4}\right)_{2},{ }^{50}$ or the $s=5 / 2$ compound $\left(\mathrm{H}_{3} \mathrm{O}\right) \mathrm{Fe}_{3}(\mathrm{OH})_{6}\left(\mathrm{SO}_{4}\right)_{2},{ }^{51}$ far fewer theoretical results are available for those higher-spin quantum numbers. In the classical limit $s \rightarrow \infty$, thermal fluctuations may lead to $\sqrt{3} \times \sqrt{3}$ long-range order as $T \rightarrow 00^{3,23}$ In most papers dealing with large-spin quantum models it has been found that quantum fluctuations select the $\sqrt{3} \times \sqrt{3}$ state. ${ }^{4,7,11,32}$
Moreover, magnetic long-range order might be possible for higher spin values. ${ }^{4,32}$

For our CCM approach for $s>1 / 2$ we use (instead of the LSUB $n$ scheme) the alternative SUB $n-m$ approximation scheme to truncate the expansions of $S$ and $\tilde{S}$ in Eqs. (3) and (4). This is because as $s$ increases the number of fundamental configuration $I$ retained at a given $\mathrm{LSUB} n$ level also increases, since each spin at any site $i$ may be raised up to $2 s$ times by its raising operator $s_{i}^{+}$, and hence each site index $i$ may be repeated up to $2 s$ times in the operators $C_{I}^{+}$of Eq. (2). In the $\mathrm{SUB} n-m$ scheme we include no more than $n$ spin flips spanning a range of no more than $m$ contiguous lattice sites. ${ }^{47,48}$ In what follows we consider the case $n=m$, i.e., $\mathrm{SUB} n-n$, which for $s=1 / 2$ is identical to the $\operatorname{LSUB} n$ scheme. Since the number of coupled ket-state equations for a certain level of SUB $n-n$ approximation increases with increasing spin quantum number $s$, the highest level of approximation we can consider is SUB8-8 for $s=1,3 / 2,2,5 / 2$, and 3 . The maximum number of ket-state equations we have to take into account is 416126 for $s=3$. For the extrapolation to $n \rightarrow \infty$ we use the same extrapolation formulas as for $s=1 / 2$, and consider the SUB $n$ - $n$ data for $n=4,5,6,7$, and 8 .

First we discuss the GS selection. In Fig. 5 we present the dependence on the anisotropy parameter $\lambda$ of the energy difference per site, $\Delta e \equiv e_{0}^{\sqrt{3} \times \sqrt{3}}-e_{0}^{q=0}$, for the highest level of approximation that we have performed (viz., SUB8-8), and for values of the spin quantum number $s=1 / 2,1, \ldots, 3$. We find that the $\sqrt{3} \times \sqrt{3}$ state is selected for all values $s>$ $1 / 2$. This is in agreement with previous studies based on an expansion around the classical limit, ${ }^{4,7,11}$ but it is in contrast to our findings for the extreme quantum case $s=1 / 2$. Hence, interestingly, our results suggest that for the frustrated quantum spin system under consideration the $s=1 / 2$ case and the cases $s>1 / 2$ may exhibit different behavior. It is interesting to note that a similar effect has also been observed for a frustrated quantum spin chain. ${ }^{52}$ We see clearly that the energy difference $\Delta e$ scaled by $s^{2}$ decreases monotonically with increasing $s$,

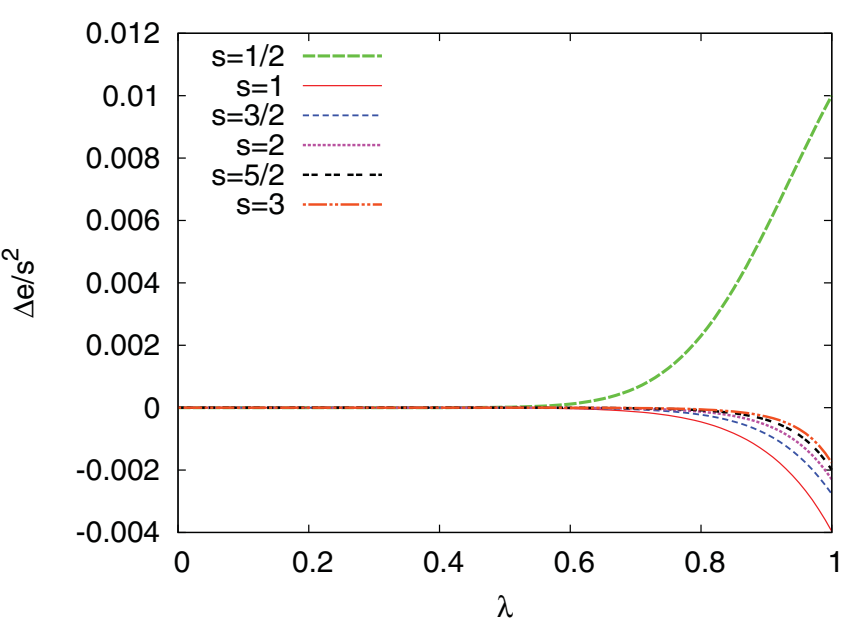

FIG. 5. (Color online) Difference of the GS energies per site, $\Delta e \equiv e_{0}^{\sqrt{3} \times \sqrt{3}}-e_{0}^{q=0}$, between the $\sqrt{3} \times \sqrt{3}$ and $q=0$ states of the kagome HAFM, calculated for the CCM SUB8-8 approximation and for spin quantum numbers $s=1 / 2,1,3 / 2,2,5 / 2$, and 3 . 


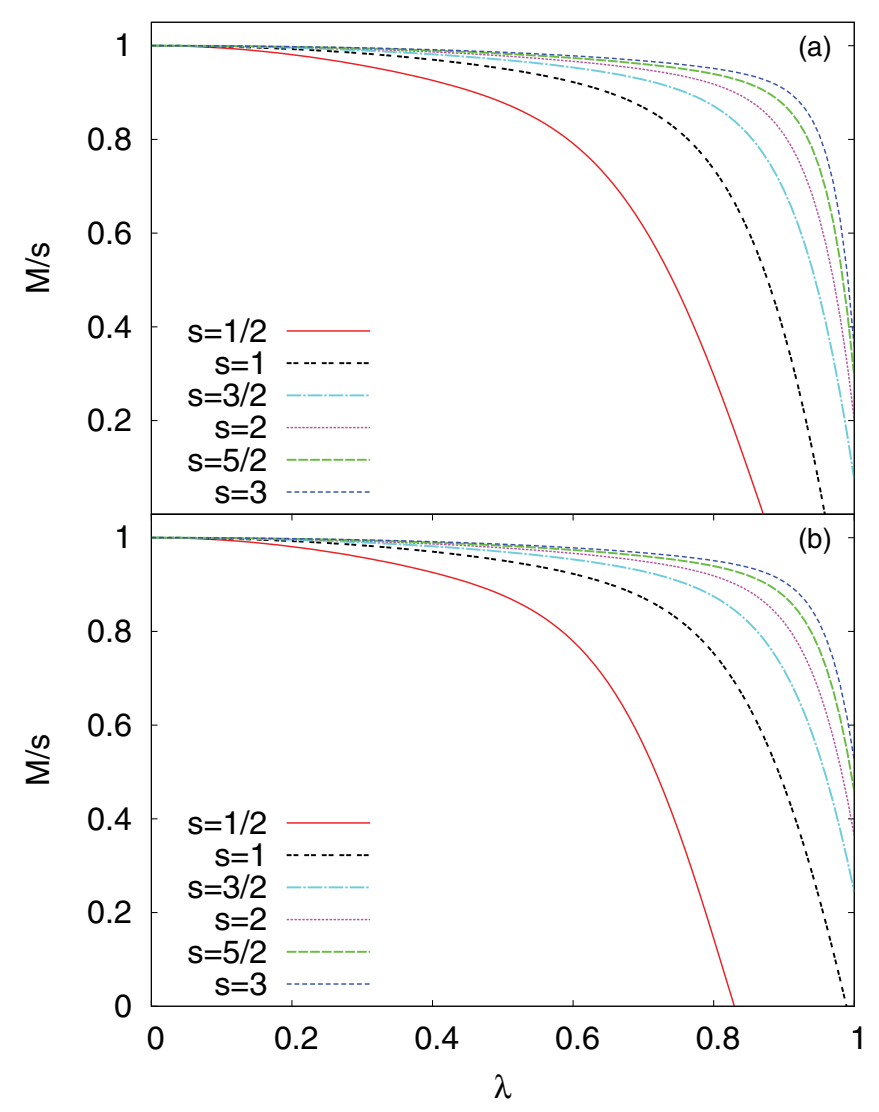

FIG. 6. (Color online) Extrapolated magnetic order parameter $M / s$ versus $\lambda$ for (a) the $\sqrt{3} \times \sqrt{3}$ reference state and (b) the $q=0$ reference state of the kagome HAFM, for various values of the spin quantum number $s$. For the extrapolations to $n \rightarrow \infty$ according to $M(n)=b_{0}+b_{1}(1 / n)^{1 / 2}+b_{2}(1 / n)^{3 / 2}$ we have used SUB $n-n$ data for $n=4,5, \ldots, 8$. [Note that even for the $s=1 / 2$ case we have excluded the (available) LSUB9 and LSUB10 data here to be consistent with the $s>1 / 2$ cases.]

thereby demonstrating that for $s \rightarrow \infty$ the $\sqrt{3} \times \sqrt{3}$ and the $q=0$ states become degenerate.

Next we discuss the magnetic order parameter $M$. To compare results for various values of $s$ it is useful to consider $M / s$. Since for $s \rightarrow \infty$ the chosen reference state is an eigenstate, we would get $M / s=1$ within our CCM approach in this classical limit. Hence, we may expect that by increasing the spin quantum number the quantity $M / s$ becomes nonzero for a certain value $s>s_{0}$ in the whole range $0 \leqslant \lambda \leqslant 1$ (see, e.g., Ref. 4). In Fig. 6 we show the extrapolated magnetic order parameter for both reference states as a function of the anisotropy parameter $\lambda$, for values $s=1 / 2,1, \ldots, 5 / 2,3$. For large values of $s$ the scaled order parameter becomes almost constant, $M / s \sim 1$, over a wide range of $\lambda$ values. However, as the true kagome point at $\lambda=1$ is approached we find a steep decay of $M / s$. Nevertheless, only for $s=1 / 2$ (as discussed already above) and for $s=1$ does the extrapolated order parameter vanish at $\lambda=1$, whereas $M / s$ remains nonzero for $s>1$. Hence, our data suggest that for higher values of $s$ a $\sqrt{3} \times \sqrt{3}$ magnetic order might be possible. To provide more detailed information on the GS properties of the kagome HAFM, we present in Table II CCM-SUB8-8 data as well
TABLE II. CCM results for the HAFM on the kagome lattice (i.e., at $\lambda=1$ ) for spin quantum numbers $s=1,3 / 2,2,5 / 2,3$. The quantity $e_{0} \equiv E / N$ is the GS energy per spin and $M$ is the magnetic order parameter (sublattice magnetization). The $\mathrm{SUB} n-n$ results are extrapolated to $n \rightarrow \infty$ according to $e_{0}(n)=a_{0}+a_{1}(1 / n)^{2}+$ $a_{2}(1 / n)^{4}$ and $M(n)=b_{0}+b_{1}(1 / n)^{1 / 2}+b_{2}(1 / n)^{3 / 2}$ using SUB $n-n$ data for $n=4,5,6,7$, and 8 .

\begin{tabular}{lcccc}
\hline \hline & \multicolumn{2}{c}{$\sqrt{3} \times \sqrt{3}$} & \multicolumn{2}{c}{$q=0$} \\
\hline$s=1$ & $e_{0} / s^{2}$ & $M / s$ & $e_{0} / s^{2}$ & $M / s$ \\
SUB8-8 & -1.383644 & 0.580079 & -1.379680 & 0.607293 \\
extr4-8 & -1.4031 & $<0$ & -1.3965 & $<0$ \\
$s=3 / 2$ & $e_{0} / s^{2}$ & $M / s$ & $e_{0} / s^{2}$ & $M / s$ \\
SUB8-8 & -1.257354 & 0.690229 & -1.254588 & 0.709167 \\
extr4-8 & -1.2680 & 0.0744 & -1.2643 & 0.2438 \\
$s=2$ & $e_{0} / s^{2}$ & $M / s$ & $e_{0} / s^{2}$ & $M / s$ \\
SUB8-8 & -1.195442 & 0.735642 & -1.193145 & 0.754580 \\
extr4-8 & -1.2026 & 0.2029 & -1.2000 & 0.3645 \\
$s=5 / 2$ & $e_{0} / s^{2}$ & $M / s$ & $e_{0} / s^{2}$ & $M / s$ \\
SUB8-8 & -1.157697 & 0.766290 & -1.155703 & 0.785822 \\
extr4-8 & -1.1627 & 0.2942 & -1.1607 & 0.4586 \\
$s=3$ & $e_{0} / s^{2}$ & $M / s$ & $e_{0} / s^{2}$ & $M / s$ \\
SUB8-8 & -1.132263 & 0.788722 & -1.130497 & 0.808862 \\
extr4-8 & -1.1360 & 0.3583 & -1.1344 & 0.5256 \\
$s \rightarrow \infty$ & $e_{0} / s^{2}$ & $M / s$ & $e_{0} / s^{2}$ & $M / s$ \\
Exact & -1 & 1 & -1 & 1 \\
\hline \hline
\end{tabular}

as extrapolated data at $\lambda=1$, for values of the spin quantum number $s=1,3 / 2,3,5 / 2$, and 3 . We see clearly that the scaled GS energy per spin approaches the classical value, $e_{0} / s^{2}=-1$, quite rapidly as $s$ is increased. On the other hand, even for the largest spin considered here (viz., $s=3$ ) the extrapolated order parameter remains relatively small, particularly for the $\sqrt{3} \times \sqrt{3}$ state.

Our CCM data for $e_{0}$ and $M$, available up to $s=3$, together with the known results in the classical limit, $\lim _{s \rightarrow \infty} e_{0} / s^{2}=$ -1 and $\lim _{s \rightarrow \infty} M / s=1$, also allow us to discuss the $s$ dependence of $e_{0} / s^{2}$ and $M / s$ in the large-s limit. In spin-wave theories one typically obtains expansions for $e_{o}$ and $M$ in powers of $1 / s \cdot{ }^{53,54}$ For the GS energy of the kagome HAFM the standard linear spin-wave theory yields for both the $\sqrt{3} \times \sqrt{3}$ and the $q=0$ states, $e_{0} / s^{2}=-1-0.4412 / s$ (see Refs. 2 and 9). On the other hand, due to the presence of the flat zero mode in the kagome HAFM, the integral for the order parameter diverges. ${ }^{9,55}$ Using an effective spin-wave theory, in which short-wavelength fluctuations are neglected, Asakawa and Suzuki ${ }^{9}$ obtained $M / s=1-0.336 / s$, whereas Chubukov ${ }^{7}$ found fluctuation corrections proportional to $s^{-2 / 3}$ (in contrast to conventional spin-waves) by using a self-consistent spinwave approach.

Here we use our extrapolated CCM data for $s=3 / 2,2,5 / 2$, and 3 to find the leading corrections to the classical values. By fitting the extrapolated GS energy $e_{0} / s^{2}$ with a fitting function $f(s)=-1+a_{1} s^{-a_{2}}$, we obtain a value for the exponent $a_{2}$ very close to one for both reference states. Hence, finally we have fitted the extrapolated CCM data for $e_{0} / s^{2}$ using $f(s)=$ $-1+x_{1} s^{-1}+x_{2} s^{-2}$. The fits yield the corresponding values $x_{1}=-0.414(-0.410)$ and $x_{2}=0.018(0.020)$ for the $\sqrt{3} \times$ $\sqrt{3}(q=0)$ reference states, as shown in Fig. 7(a). Obviously, 

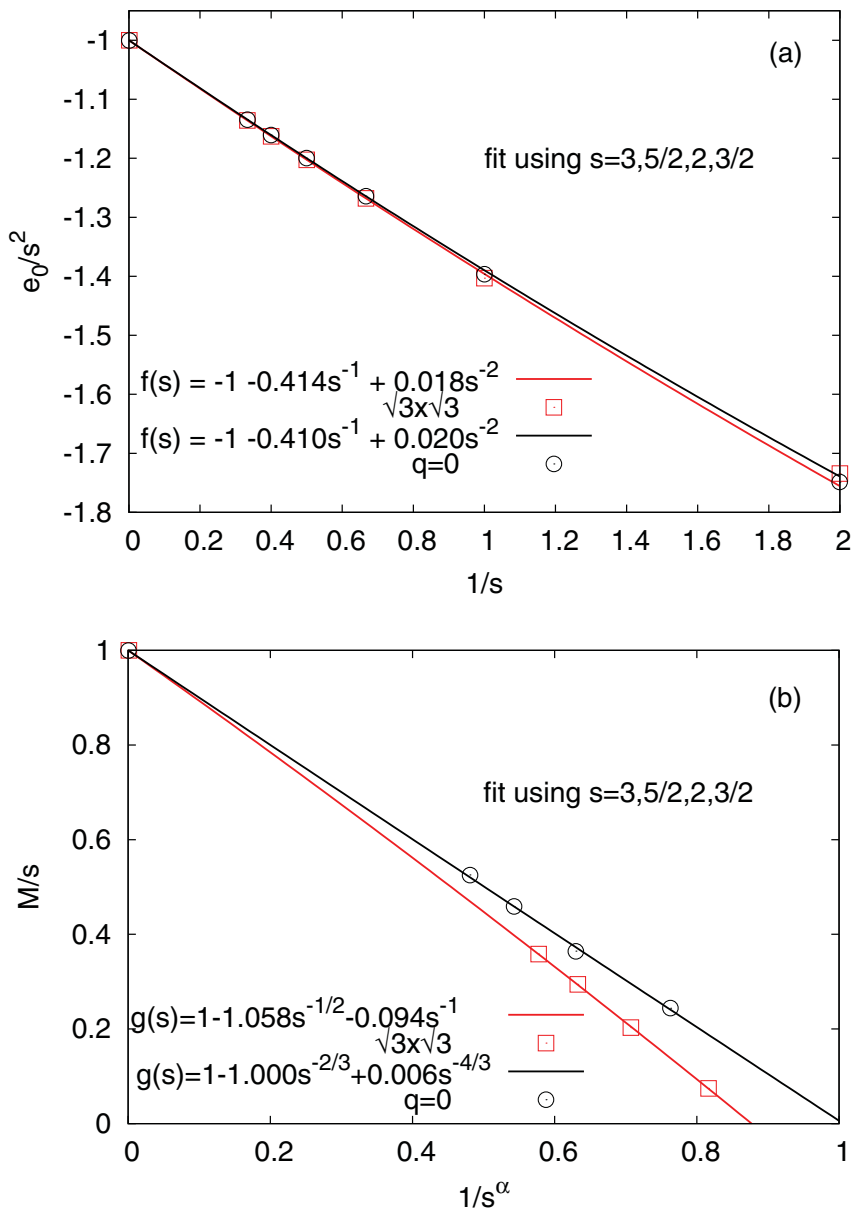

FIG. 7. (Color online) Dependence on the spin quantum number $s$ of (a) the extrapolated scaled GS energy per spin, $e_{0} / s^{2}$, and (b) the extrapolated scaled magnetic order parameter, $M / s$, of the kagome HAFM. For $M / s$ the exponent $\alpha=1 / 2$ for the $\sqrt{3} \times \sqrt{3}$ state and $\alpha=2 / 3$ for the $q=0$ state. (Symbols are CCM data points; lines are fits to the data points).

the $\frac{1}{s}$ prefactor is close to that of the linear spin-wave theory, and the contribution of the next order is small. Note, however, that if the fitting function $f(s)$, with the above given values for $x_{1}$ and $x_{2}$, is applied to the $s=1 / 2$ case, it does not reproduce the GS selection of the $q=0$ state in this extreme quantum case [c.f., Fig. 7(a) at the value $1 / s=2$ ].

Next we use $g(s)=1+b_{1} s^{-b_{2}}$ as a fitting function to fit the extrapolated CCM order parameters $M / s$, again using data or $s=3 / 2,2,5 / 2$, and 3 . For the exponent $b_{2}$ we get the values $b_{2}=0.529$ for the $\sqrt{3} \times \sqrt{3}$ reference state and $b_{2}=0.666$ for the $q=0$ reference state. Clearly, the leading correction is not proportional to $s^{-1}$; rather
Chubukov's result ${ }^{7}$ of a leading correction proportional to $s^{-2 / 3}$ is confirmed for the $q=0$ state. By contrast, for the $\sqrt{3} \times \sqrt{3}$ reference state our results are in favor of a leading correction for the order parameter proportional to $s^{-1 / 2}$. Hence, finally we have fitted the extrapolated CCM data for $M / s$ using the fitting functions $g(s)=1+y_{1} s^{-1 / 2}+$ $y_{2} s^{-1}\left[g(s)=1+y_{1} s^{-2 / 3}+y_{2} s^{-4 / 3}\right]$ for the $\sqrt{3} \times \sqrt{3}[q=$ $0]$ reference states. The fits yield the corresponding values $y_{1}=-1.058[-1.000]$ and $y_{2}=-0.094$ [0.006], as shown in Fig. 7(b).

\section{SUMMARY}

In the present investigation we present data for the GS energy per spin, $e_{0}$, and the order parameter (sublattice magnetization), $M$, of the kagome HAFM for spin quantum numbers $s=1 / 2,1,3 / 2,2,5 / 2$, and 3 , using high-order CCM-SUB $n-n$ calculations based on the $\sqrt{3} \times \sqrt{3}$ and the $q=0$ reference states. Our best estimate of the GS energy for the $s=1 / 2$ case, viz., $e_{0}=-0.4372$, is clearly below rigorous upper bounds reported recently, ${ }^{27,28}$ and it also agrees well with recent accurate $\mathrm{DMRG}^{28}$ and $\mathrm{ED}^{29}$ results. We find that the GS selection by quantum fluctuations depends on the spin quantum number $s$. While for $s=1 / 2$ the $q=0$ state is selected, for all values $s>1 / 2$ the $\sqrt{3} \times \sqrt{3}$ state has lower energy. Using CCM data for $s=3 / 2,2,5 / 2$ and 3 we determine also the leading quantum corrections to the classical values of the GS energy and the order parameter.

For the order parameter $M / s$ obtained by an appropriate extrapolation of the CCM-SUB $n-n$ data we get small (but nonzero) values $M / s>0$ for all values of the spin quantum number $s>1$. For $s=1 / 2$ and $s=1$ the situation is different. Clearly, in any SUB $n-n$ approximation for finite $n$ we have a magnetic quantum ground state (i.e., a finite $M / s$ ) with the corresponding symmetry of the classical ground state. Extrapolating to $n \rightarrow \infty$ we get a nonmagnetic ground state (vanishing order parameter) but locally the magnetic correlations still fit to the symmetry of the classical ground state. However, they can be extremely short ranged; i.e., the ground state can be considered as a spin-liquid state with spin correlations on a very short-ranged length scale. However, the true kagome ground state might have also an admixture of contributions of other classical ground states renormalized by quantum fluctuations.

\section{ACKNOWLEDGMENTS}

O.G. and J.R. thank R. Zinke and J. Schulenburg for fruitful discussions.
${ }^{1}$ J. B. Marston and C. Zeng, J. Appl. Phys. 69, 5962 (1991).

${ }^{2}$ A. B. Harris, C. Kallin, and A. J. Berlinsky, Phys. Rev. B 45, 2899 (1992).

${ }^{3}$ D. A. Huse and A. D. Rutenberg, Phys. Rev. B 45, 7536 (1992).

${ }^{4}$ S. Sachdev, Phys. Rev. B 45, 12377 (1992).
${ }^{5}$ J. T. Chalker and J. F. G. Eastmond, Phys. Rev. B 46, 14201 (1992).

${ }^{6}$ R. R. P. Singh and D. A. Huse, Phys. Rev. Lett. 68, 1766 (1992).

${ }^{7}$ A. Chubukov, Phys. Rev. Lett. 69, 832 (1992).

${ }^{8}$ P. W. Leung and V. Elser, Phys. Rev. B 47, 5459 (1993).

${ }^{9}$ H. Asakawa and M. Suzuki, Physica A 205, 687 (1994). 
${ }^{10}$ C. Zeng and V. Elser, Phys. Rev. B 51, 8318 (1995).

${ }^{11}$ C. L. Henley and E. P. Chan, J. Magn. Magn. Mater. 140-144, 1693 (1995).

${ }^{12}$ C. Waldtmann, H.-U. Everts, B. Bernu, C. Lhuillier, P. Sindzingre, P. Lecheminant, and L. Pierre, Eur. Phys. J. B 2, 501 (1998).

${ }^{13}$ M. Mambrini and F. Mila, Eur. Phys. J. B 17, 651 (2000).

${ }^{14}$ D. J. J. Farnell, R. F. Bishop, and K. A. Gernoth, Phys. Rev. B 63, 220402 (2001).

${ }^{15}$ B. H. Bernhard, B. Canals, and C. Lacroix, Phys. Rev. B 66, 104424 (2002).

${ }^{16}$ P. Nikolic and T. Senthil, Phys. Rev. B 68, 214415 (2003).

${ }^{17}$ D. Schmalfuß, J. Richter, and D. Ihle, Phys. Rev. B 70, 184412 (2004).

${ }^{18}$ R. Budnik and A. Auerbach, Phys. Rev. Lett. 93, 187205 (2004).

${ }^{19}$ S. Capponi, A. Läuchli, and M. Mambrini, Phys. Rev. B 70, 104424 (2004).

${ }^{20}$ R. R. P. Singh and D. A. Huse, Phys. Rev. B 76, 180407(R) (2007).

${ }^{21}$ R. R. P. Singh and D. A. Huse, Phys. Rev. B 77, 144415 (2008).

${ }^{22}$ H. C. Jiang, Z. Y. Weng, and D. N. Sheng, Phys. Rev. Lett. 101, 117203 (2008).

${ }^{23}$ C. L. Henley, Phys. Rev. B 80, 180401(R) (2009).

${ }^{24}$ P. Sindzingre and C. Lhuillier, Europhys. Lett. 88, 27009 (2009).

${ }^{25}$ D. Poilblanc, M. Mambrini, and D. Schwandt, Phys. Rev. B 81, 180402 (2010).

${ }^{26}$ R. F. Bishop, P. H. Y. Li, D. J. J. Farnell, and C. E. Campbell, Phys. Rev. B 82, 104406 (2010).

${ }^{27}$ G. Evenbly and G. Vidal, Phys. Rev. Lett. 104, 187203 (2010).

${ }^{28}$ S. Yan, D. A. Huse, and S. R. White, Science 332, 1173 (2011); see also e-print arXiv:1011.6114.

${ }^{29}$ A. M. Läuchli, J. Sudan, and E. S. Sørensen, Phys. Rev. B 83, 212401 (2011).

${ }^{30}$ Y.-M. Lu, Y. Ran, and P. A. Lee, Phys. Rev. B 83, 224413 (2011).

${ }^{31}$ H. Nakano and T. Sakai, J. Phys. Soc. Jpn. 80, 053704 (2011).

${ }^{32}$ O. Cépas and A. Ralko, Phys. Rev. B 84, 020413 (2011).

${ }^{33}$ T. Tay and O. I. Motrunich, Phys. Rev. B 84, 020404(R) (2011).

${ }^{34}$ Y. Iqbal, F. Becca, and D. Poilblanc, Phys. Rev. B 84, 020407(R) (2011).

${ }^{35}$ R. Darradi, J. Richter, and D. J. J. Farnell, Phys. Rev. B 72, 104425 (2005).

${ }^{36}$ D. Schmalfuß, R. Darradi, J. Richter, J. Schulenburg, and D. Ihle, Phys. Rev. Lett. 97, 157201 (2006).
${ }^{37}$ R. Darradi, O. Derzhko, R. Zinke, J. Schulenburg, S. E. Krüger, and J. Richter, Phys. Rev. B 78, 214415 (2008).

${ }^{38}$ R. F. Bishop, P. H. Y. Li, R. Darradi, and J. Richter, J. Phys. Condens. Matter 20, 255251 (2008).

${ }^{39}$ D. J. J. Farnell, R. Zinke, J. Schulenburg, and J. Richter, J. Phys. Condens. Matter 21, 406002 (2009).

${ }^{40}$ J. Richter, R. Darradi, J. Schulenburg, D. J. J. Farnell, and H. Rosner, Phys. Rev. B 81, 174429 (2010).

${ }^{41}$ D. J. J. Farnell, R. F. Bishop, P. H. Y. Li, J. Richter, and C. E. Campbell, Phys. Rev. B 84, 012403 (2011).

${ }^{42}$ D. J. J. Farnell, R. Darradi, R. Schmidt, and J. Richter, Phys. Rev. B 84, 104406 (2011).

${ }^{43}$ C. Zeng, D. J. J. Farnell, and R. F. Bishop, J. Stat. Phys. 90, 327 (1998).

${ }^{44}$ R. F. Bishop, in Microscopic Quantum Many-Body Theories and Their Applications, edited by J. Navarro and A. Polls, Lecture Notes in Physics, Vol. 51 (Springer, Berlin, 1998), p.1.

${ }^{45}$ R. F. Bishop, D. J. J. Farnell, and C. Zeng, Phys. Rev. B 59, 1000 (1999).

${ }^{46}$ R. F. Bishop, D. J. J. Farnell, S. E. Krüger, J. B. Parkinson, J. Richter, and C. Zeng, J. Phys. Condens. Matter 12, 6887 (2000).

${ }^{47}$ D. J. J. Farnell, R. F. Bishop, and K. A. Gernoth, J. Stat. Phys. 108, 401 (2000).

${ }^{48}$ D. J. J. Farnell and R. F. Bishop, in Quantum Magnetism, edited by U. Schollwöck, J. Richter, D. J. J. Farnell, and R. F. Bishop, Lecture Notes in Physics, Vol. 645 (Springer, Berlin, 2004), p. 307.

${ }^{49}$ For the numerical calculation we use the program package "The crystallographic CCM" (D. J. J. Farnell and J. Schulenburg).

${ }^{50}$ A. Keren, K. Kojima, L. P. Le, G. M. Luke, W. D. Wu, Y. J. Uemura, M. Takano, H. Dabkowska, and M. J. P. Gingras, Phys. Rev. B 53, 6451 (1996); S.-H. Lee, C. Broholm, M. F. Collins, L. Heller, A. P. Ramirez, Ch. Kloc, E. Bucher, R. W. Erwin, and N. Lacevic, ibid. 56, 8091 (1997).

${ }^{51}$ A. S. Wills, A. Harrison, C. Ritter, and R. I. Smith, Phys. Rev. B 61, 6156 (2000).

${ }^{52}$ D. V. Dmitriev, V. Ya. Krivnov, and J. Richter, Phys. Rev. B 75, 014424 (2007)

${ }^{53}$ C. J. Hamer, Z. Weihong, and P. Arndt, Phys. Rev. B 46, 6276 (1992).

${ }^{54}$ A. V. Chubukov, S. Sachdev, and T. Senthil, J. Phys. Condens. Matter 6, 8891 (1994).

${ }^{55}$ J. Richter, J. Schulenburg, A. Honecker, and D. Schmalfuß, Phys. Rev. B 70, 174454 (2004). 